\title{
Development of 3D Printed Bone Reduction with Fracture Lines in Different Color
}

\author{
Irwansyah Idram ${ }^{1}$, Yu-Wen Tseng ${ }^{1}$, Jiing-Yih Lai ${ }^{1 *}$, Chao-Yaug Liao ${ }^{1}$, Pei-Yuan \\ Lee $^{2}$ \\ ${ }^{1}$ Department of Mechanical Engineering, National Central University, Taoyuan, Taiwan \\ ${ }^{2}$ Department of Orthopedic, Show Chwan Memorial Hospital, Changhua, Taiwan \\ irwansyah@unsyiah.ac.id, uwen1002@gmail.com, jylai@ncu.edu.tw, \\ cyliao@ncu.edu.tw,b1208b@ms26.hinet.net
}

\begin{abstract}
The bone fragment and fracture lines may not clear enough to be visualized after bone reduction and may be hidden behind other bones. A 3D printing technology can be used to deal with this drawback. A series of algorithms were developed to ensure that two adjacent bone fragments fabricated can be assembled successfully. In this study, we developed a bone reduction algorithm, and a mesh-overlapping detection and removal algorithm to ensure that two 3D-printed fragments are easily assembled and disassembled. In addition, we also developed a method to detect and extract fracture boundary of two adjacent fragments, and then apply a multi-color 3D printing technology to fabricate the assembled bone fragments. The advantage of the proposed method is that we can show the status of 3D-printed bone fragments before and after bone reduction, with fracture lines printed in different color.
\end{abstract}

\section{Introduction}

In the preoperative planning surgery, it is important to understand the bone fracture site and level of fracture damaging. The fracture pattern of a comminuted bone fractures is not yet thoroughly understood because of its complex geometry. The bone fragments would be hidden behind other bones, and hence cannot be visualized after bone reduction. 3D printing technology can be used to overcome the limitation of medical imaging data and present realistic anatomical bone fracture (Yang, et al., 2016). The 3D-printed models with different color fragments can also be used to assist clinician-patient communication, and medical students to understand specific details of the patients fracture such as type of fractures, number of fragments,

*Corresponding author: Jiing-Yih Lai, Department of Mechanical Engineering, National Central University, No. 300, Zhongda Road, Zhongli, Taoyuan City, 320, Taiwan. Tel.: +886-3-4227151; Fax:+886-3-4226062; E-mail: jylai@ncu.edu.tw 
articular surface fractures and fracture lines direction (Huang, et al., 2018). A full-scale 3D printed model with a different color is fabricated to distinguish anatomical parts and show critically damaged fracture fragments (Chen, et al., 2017). In this study, we developed a series of algorithms to ensure that two adjacent bone fragments fabricated using 3D printing can be assembled successfully. A bone reduction algorithm and a mesh-overlapping detection and removal algorithm were developed to ensure that two 3D-printed fragments can easily be assembled and disassembled. In addition, we also developed a method to detect and extract fracture boundary of two adjacent fragments, and then apply a multi-color 3D printing technology to fabricate the assembled bone fragments. The advantage of the proposed method is that we can show the status of 3D-printed bone fragments before and after bone reduction, with fracture lines printed in different color.

\section{Materials and Methods}

A 3D model of the simple fracture acetabular was reconstructed directly from CT scan images. A single fracture line separated the acetabulum through both posterior and anterior columns becoming two or three bony fragments. Each of the fragments can be manipulated individually during the bone reduction process. The acetabular fragments were segmented and restored back into their original anatomy position using a semi-automatic bone reduction algorithm. The surface contact of two adjacent bone fragments after bone reduction was isolated by using an overlapping removal algorithm. The algorithm began with repositioning the bone fragments (F1) and (F2). Let M and L represent two set of fragment data with $\mathrm{mi}=(\mathrm{m} 1, \mathrm{~m} 2 \ldots \mathrm{mn})$ and $\mathrm{lj}=(11,12 \ldots \mathrm{ln})$. Then, the axis alignment bounding box (AABB) method was employed to detect mesh intersection between two adjacent fragments. Since the intersection bounding box (IBB) was calculated, the overlapping meshes might exist on the intersected boundary boxes. The algorithm for boundary boxes subdivisions was also applied to check the triangular meshes of both models for each IBB. Then, a cell subdivision algorithm was employed to divide an initial boundary box rapidly, which covers the entire set of triangular meshes and performs iteratively. The bone fragment model was converted to solid models and exported to a **. STL (STereoLithography) file using a CAD program before sending to the 3D printing machine. The solid model with certain shell thickness were attained by offsetting the triangles or vertices directly along the normal directions. Then, the Boolean operation was used to merge and subtract the intersection regions of bone fragment surfaces (Materialise Magics 13). The printing process of bone fragments was simulated using a Fused Deposition Modelling (FDM) default software. A multi-color 3D printer (Ultimaker Dual Extrusion Print Head), a FDM-based 3D printer that offers two PolyLactic Acid (PLA) printed-color materials, built a full scale of acetabulum fracture.

\section{Results}

The results of extracted fractured bone regions and fracture lines boundary of a transverse acetabulum fracture (B1-1) are shown in Fig. 1. The mesh overlapping detection algorithm has shown the viable result to identify the surface fracture region. The fracture bone meshes on the contact surface region were classified within three mesh regions. The inner fracture-line region (red color) represents the fracture area for both fragments. The outer fracture-line region (black color) indicates the unbroken bone. The fracture lines of fragment boundary (green color) were obtained by expanding a single layer mesh of the inner fracture region. Figs. 1(a) - (c) depict actual 3D models of an acetabular fracture, bone fragment after reduction and bone fracture surface regions, respectively. The surface bones fracture regions were isolated and converted to a solid model, as shown in Fig. 1 (d). 


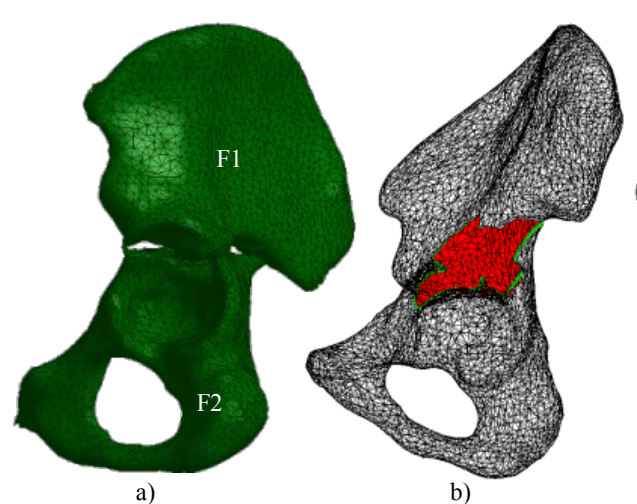

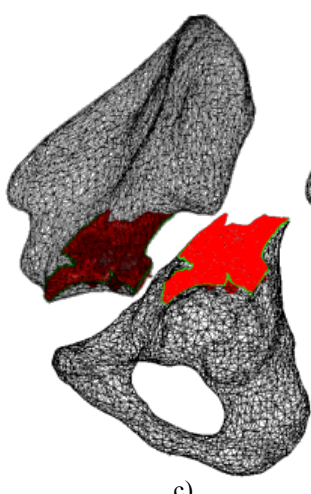

c)

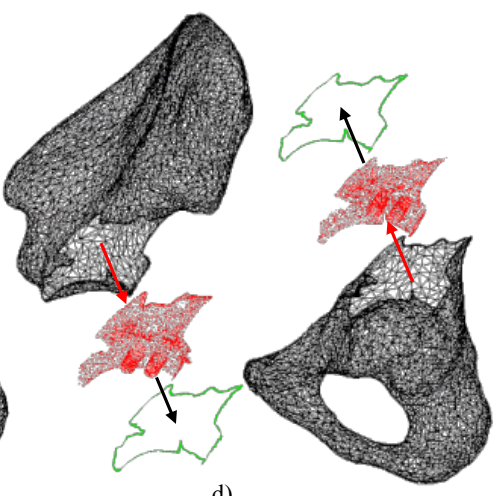

d)

Figure 1: 3D model reconstruction and bone fracture boundary.

The real size of transverse acetabular fracture was fabricated within $4 \sim 12 \mathrm{~h}$, depending on the anatomical part. Fig. 2 presents the results of 3D printing acetabular with transverse (B1-1) and T-type (B2) fractures. The original 3D model, after bone reduction, and 3D-printed part without and processing for B1-1 fracture are shown in Figs. 2(a) - (c), respectively, and Figs. 2(f) - (h) show the same results for $\mathrm{B} 2$ fracture. The result after repositioning and removing overlapped meshes using our algorithm is presented in Figs. 2(d) (3D model using our algorithm) and (e) (3D-printed part) for B1-1 fracture and Figs. 2(i) and (j) for B2 fracture. Then, Figs. 2(k) - (m) present the 3D printed bone with fracture fragment boundary printed in a different color.

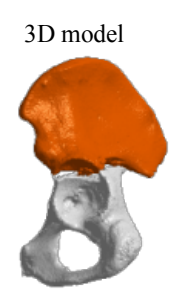

(a)

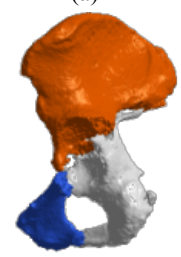

(f)

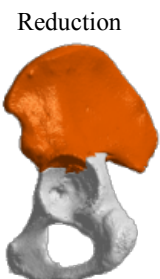

(b)

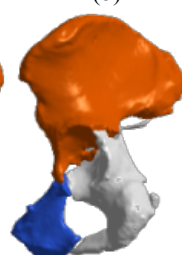

(g)
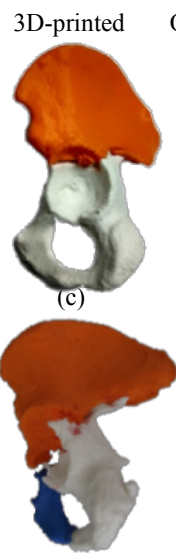

(h)

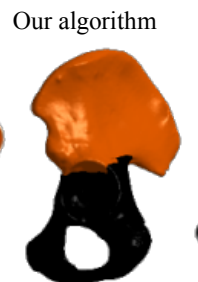

(d)

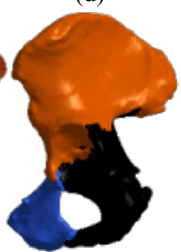

(i)

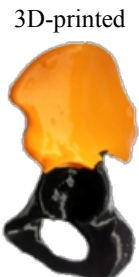

(e)

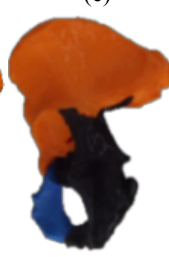

(j)

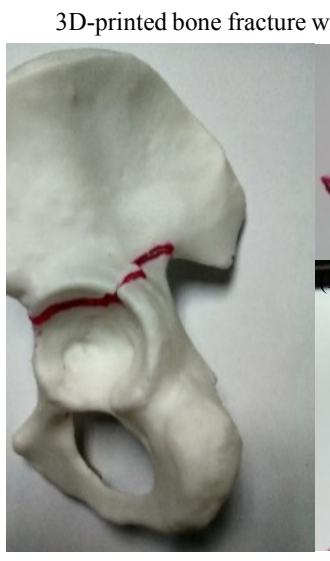

(k)

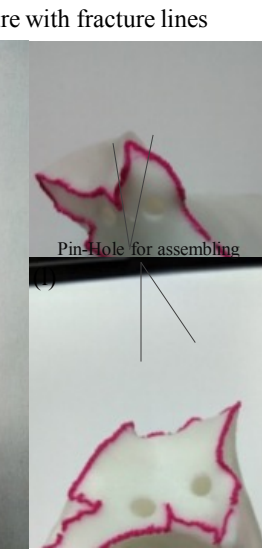

(m)

Figure 2: 3D printed replicas of the transverse acetabular fracture (a) and T-type fracture (f).

It can be recognized that the fracture lines split the surface fractures region and un-fractured area. The thickness of fracture lines along the fragments boundary is approximately $0.5 \mathrm{~mm}$. The surface fracture regions were painted referring to 3D-printing material filament color. In this study, the white color was addressed to ilium and ischium parts, red was chosen for the fracture lines and white color for the contact surface area. To evaluate the successful assembly of the bone fragments, we measured the particular gap error of the assembled 3D-printed bone segments along the joint area. The minimum and maximum gap errors at each point measurement were within the range $0.63-1.89 \mathrm{~mm}$. 


\section{Discussion}

In this study, we showed that the proposed algorithms have successfully overcome the assembled and disassembled limitation on fracture bone models obtained from CT images. When the two displaced fragments models were recovered from their original anatomy position in a virtual bone reduction process, overlapping and gap errors existed influencing the assembly of 3D-printed bone fragments (Irwansyah, Redyarsa, Lai, Essomba, \& Lee, 2017). The meshes detection and removal algorithm was able to check mesh intersection and remove the overlapping meshes. Then, the meshes-isolating algorithm specified the fracture surface area of adjacent bone fragments, for extracting the fracture lines, regions inside fracture lines, and regions outside fracture lines. The smoothing process was also employed to remove the interference from the meshes defect so that the 3D-printed bone fragments with fracture lines printed in different color can be assembled well. The gap errors along the surface contact of the assembled 3D-printed bone segments were measured and the maximum error was less than $2 \mathrm{~mm}$; the discrepancy error should be as small as possible. A 3D-printed bone fracture with a different color on surface fracture area was printed using high-end full color 3D printer to imitate the realistic anatomy fracture (Bizzotto, et al., 2015) and (Bizzotto, et al., 2016). The 3D-printed bone offers 1:1 in scale as a reference in pre-surgery, so that the surgeon can observe the distribution of the fracture line in 3D space, which is usually difficult to achieve by using a computer 3D model. In our algorithm, we provided a technique to print the fracture lines along the fracture boundary of each 3Dprinted bone fragment and emphasized particularly on the assembly and disassembly of the bone fragments. In addition, we exploited the feasibility to print the fracture bone and fracture lines in different color using low-end 3D printer. Eventhough, in a simple fracture case the 3D printed bone with fracture lines may not be significantly helpful, but in the complex displaced fractures may useful to assists the surgeon, resident, and patient getting specific information of bone fractures.

\section{References}

Bizzotto, N., Sandri, A., Regis, D., Romani, D., Tami, I., \& Magnan, B. (2015). Three-dimensional printing of bone fractures: A new tangible realistic way for preoperative planning and education. Surgical Innovation, 548-551. Retrieved from https://www.ncbi.nlm.nih.gov/pub med/25646008

Bizzotto, N., Tami, I., Santucci, A., Adani, R., Poggi, P., Romani, D., \& Magnan, B. (2016). 3D printed replica of articular fractures for surgical planning and patient consent: a two years multi-centric experience. $3 D$ printing in medicine, 1-6. Retrieved from https://link. springer.com /article/10.1186\%2Fs41205-016-0006-8

Chen, S., Pan, Z., Wu, Y., Gu, Z., Li, M., Liang, Z., \& Pan, H. (2017). The role of three-dimensional printed models of skull in anatomy education: a randomized controlled trail. Scientific Reports, 1-11. Retrieved from https://www.ncbi.nlm.nih.gov/pubmed/28373643

Huang, Z., Song, W., Zhang, Y., Zhang, Q., Zhou, D., Zhou, X., \& He, Y. (2018). Three-dimensional printing model improves morphological understanding in acetabular fracture learning: A multicenter, randomized, control study. PLoS ONE, 1-2. Retrieved from https://www.ncbi. nlm.nih.gov/pubmed/29342198

Irwansyah, Redyarsa, D.B., Lai, J.Y., Essomba, T., \& Lee, P.Y. (2017). Integration of computer-aided pre-operative planning and $3 \mathrm{D}$ printing technology for comminuted fracture bone surgery. IEEE International Conference on Applied System Innovation (pp. 1235-1238). Sapporo, Japan: IEEE Xplore. Retrieved from https://ieeexplore.ieee.org/document/7988116/

Yang, L., Shang, X.W., Fan, J.N., He, Z.X., Wang, J.J., Liu, M., \& Ye, C. (2016). Application of 3D printing in the surgical planning of trimalleoar fracture and doctor-patient communication. BioMed Research, 1-5. Retrieved from https://www.hindawi.com/journals/bmri /2016/2482086/ 